\title{
Structural and computational investigation of an imine-based propeller-shaped macrocyclic cage
}

\author{
Sriram Srinivasa Raghvan ${ }^{1}$ (1) $\cdot$ Suresh Madhu $^{2,3} \cdot$ Velmurugan Devadasan $^{1} \cdot$ Gunasekaran Krishnasamy $^{1}$
}

Received: 12 August 2020 / Accepted: 20 January 2021 / Published online: 28 January 2021

(c) The Author(s) 2021 OPEN

\begin{abstract}
In this study, we present the synthesis, spectroscopic and structural characterization of self-assembling gem-dimethyl imine based molecular cage (IMC). Self-assembling macrocycles and cages have well-defined cavities and have extensive functionalities ranging from energy storage, liquid crystals, and catalysts to water splitting photo absorber. IMC has large voids i.e., $25 \%$ of the total crystal volume thus could accommodate wide substrates. The synthesized imine-based molecular cages are stabilized by coaxial $\pi$ bonded networks and long-range periodic van der Waal and non-bonded contacts as observed from the crystal structure. IMC also has typical properties of soft condensed matter materials, hence theoretical prediction of stress and strain tensor along with thermophysical properties were computed on crystal system and were found to be stable. Molecular dynamics revealed IMC is stabilized by, strong interactions between the interstitial phenyl rings. Density functional theory (DFT) based physicochemical properties were evaluated and has band gap of around $2.38 \mathrm{ev}(520 \mathrm{~nm})$ similar to various photocatalytic band gap materials.
\end{abstract}

Keywords Gem-dimethyl · Macrocyclic cage · Thermophysical analysis · Morphology · Photophysics

\section{Introduction}

Macrocyclic compounds generally feature multiple rings or caged structures [1]. Organic macrocyclic cages and related coordinated complexes have shown wide range of applications from molecular catalysis to gas storage devices [2]. The robustness and flexibility to accommodate large substrates improve their utility in storage applications [3]. The spatial and temporal geometry of macrocyclic cages are important to accommodate various substrates. Coordination with different metal ions leads to the formation of metallosupramolecules exhibiting excellent host-guest interactions [4]. Imine-based molecular cages have favorable physicochemical properties as conductive polymers [5, 6]. Porphyrin-based photoabsorber has unique band gap width by virtue of heterocyclic conjugated system [7]. The presence of oxygen in photoabsorber architecture is known to induce triplet excitation and hence reduces the degree of electron delocalization $[8,9]$. Imine-based macrocycles require molecular robustness, as they are prone to hydrolysis in acidic environment leading to undesirable oligomeric polymorphism either cyclic or acyclic. Template-derived self-assembling macrocyclic cages offer potential commercial applications

Sriram Srinivasa Raghvan, Suresh Madhu contributed equally to this work.

Supplementary information The online version contains supplementary material available at (https://doi.org/10.1007/s42452-021-04255 -7).

$\triangle$ Sriram Srinivasa Raghvan, hypowergravity@gmail.com; $\bowtie$ Suresh Madhu, chemistrysuresh1986@gmail.com | ${ }^{1}$ Department of Crystallography and Biophysics, University of Madras, Chennai, Tamilnadu 600025, India. ${ }^{2}$ Division of Organic Chemistry, CSIR-National Chemical Laboratory, Dr. Homi Bhabha Road, Pune, Maharashtra 411008 , India. ${ }^{3}$ Academy of Scientific and Innovative Research (AcSIR), Ghaziabad 201 002, India. 
as photoabsorber. The caged organic framework having higher spatial occupancy with $\pi$-conjugated systems has many adjunct functions in the area of catalysis and gas adsorptions. Metal-organic frameworks having appropriate band gap and molecular geometry can also be used for water splitting applications $[2,10,11]$.

Physicochemical, thermo-physical, molecular elasticity and stress-strain tensor are important parameters to design a robust metal organic framework to realize macroscale applications. Physicochemical parameters of photoabsorber directs activation energy, energy gap and chemical hardness of the system. Elasticity analysis will reveal the relative pressure limit that the system can withstand without volumetric deformations. Stress and strain analysis also predicts the molecular robustness, stability and compressions.

The coordination of the macrocycle with metals generally induces polymorphisms in crystals. One of the main steps in water splitting is the oxidation of water, which is both thermodynamically and kinetically demanding. Effective catalysis lowers the kinetic barrier thereby enhances the rate of oxygen production. In this regard, transition metal ions and oxides [such as $\mathrm{TIO}_{2} \mathrm{Mn}^{2+}, \mathrm{Ru}^{2+}$, etc.,.] are generally characterized as useful catalytic elements [12]. Metal-organic frameworks (MOF) with bigger macrocyclic cages having higher aromaticity and tend to have self-excitation properties. Also, it is necessary to create macrocyclic structures having desirable physiochemical properties. Most of the studied compounds have macrocyclic tetrapyrroles groups [13]. Numerous energy harnessing molecules are macrocyclic in nature, like chlorophyll made of porphyrins [14]. Recently, graphene has been proposed to have superconductivity [15] and is believed to be useful for water splitting applications if a macrocyclic architecture is suitably incorporated [16]. Hence, structural analysis of macrocyclic cages becomes important to design above mentioned useful frameworks. Along with conformational features, intermolecular arrangement and molecular interactions play a crucial role in the packing of molecules in crystalline materials having desired physiochemical properties [17].

Self-assembling macrocyclic cages are particularly quite useful as they require reduced number of user-derived inputs for conformational assembly [18]. The synthesized gem-dimethyl based cage that has been analysed in this work tends to have self-assembling characteristics. In that direction, this report explains the structural description of gem-dimethyl imine based macrocyclic cage, followed by FTIR and NMR characterization. Simulation of IMC crystal with unit cell, dimension of $2 \times 2 \times 2$ were taken to evaluate thermophysical and mechanical properties. Density functional theory based physicochemical properties were also evaluated to identify the electronic distribution of gemdimethyl based imine-based macrocyclic cage for photocatalytic like application.

\section{Results and discussion}

The crystal structure of imine-based macrocyclic cage (IMC) (Fig. 1a) shows the presence of three molecules in the asymmetric unit with three-fold symmetry. Crystal data and structure determination details are listed (Table 1 and S1). The IMC acquires macromolecular tricyclic arrangement to form a propeller-shaped architecture with three paddles $A, B$ and $C$ linking the central triphenylamine moieties to form a cage structure (Fig. 1b). The displacement ellipsoid plot for all the three cages
Fig. 1 Representation of imine based macrocyclic cage. a Chemical representation of IMC, b Stick representation of IMC having propeller-shaped architecture having three paddles $A, B$ and $C$, c Thermal ellipsoid plot ( $50 \%$ probability) of a symmetric unit of nonhydrogen atoms (a)

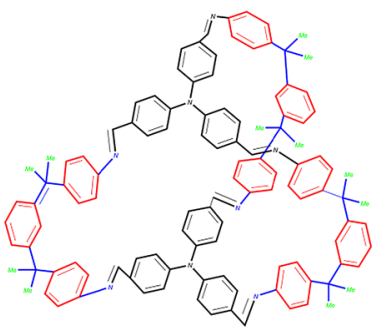

(b)

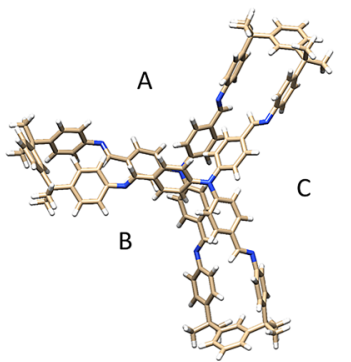

(c)

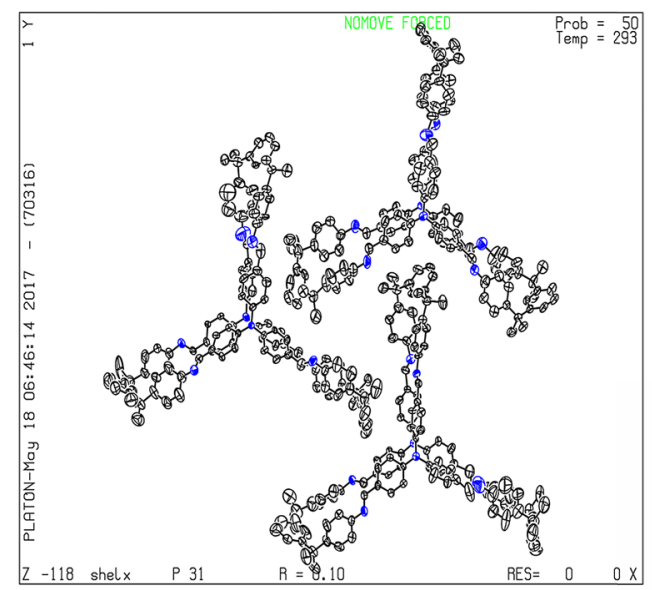


with $50 \%$ probability is shown in Fig. 1c. For clarity, IMC molecules with the numbering scheme are shown in Figure S1. Paddles with five aromatic rings and two gem-dimethyl methyl groups make them hydrophobic. This architecture which consists of two triphenylamine moieties [average distance between nitrogen atoms $(N x \ldots N y=5.17 \AA)$ [Table S2, Figure S 2] connected by three gem-dimethyl groups through imine linker provide a void within the cage. Three phenyl rings connected to the central Nitrogen are tilted relative to one another to avoid steric hindrance. Fifteen IMC molecules are packed in a triclinic unit cell having a $\underline{3}_{1}$ screw component along the $Z$ direction with molecule placed at $1 / 2$ and $1 / 3$ translations.

A total of 15 IMC molecules can be packed in a unit cell which measures around $7 \mathrm{~nm}$ wide. The crystal packing is stabilized by T-Shaped $\pi-\pi$ interactions along with parallel displaced van der Waals intermolecular interactions. The unit cell of IMC packed with the molecule is shown in Figure S7. Some of the main interatomic contacts which stabilize the packing of IMC are listed in Table S3 along with symmetry codes. Packing and structure of IMC are stabilized by week $\pi-\pi$ interactions. The centroid distance of phenyl moiety in IMC is given in Table S4, (Figure S3). Contact map of IMC and its molecular packing interaction patterns are shown in Figure S4. The methyl groups also show interactions with phenyl ring through methyl- $\pi$ interactions. All the gem-dimethyl groups are held closely (with an average distance of $2.3 \AA$ ) which reveal the compactness of the IMC system (in the crystal) as shown by the distance map (Figure S5). The contact surface and void space were analyzed with the probe radius $1.2 \AA$ as shown in Figure $S 6$. Void volume in the crystal is calculated to be $25 \%$ of the total crystal volume. From the experimental i.e. from the crystal structure, the volume of the crystal is $23896.25 \AA^{3}$ and the void volume is $6197.03 \AA^{3}$.

Fig. 2 IMC molecules in unit cell along with unit cell packing of order $2 \times 2 \times 2$ of molecules having centroid fit

\subsection{FT-IR and NMR analysis}

IMC molecular crystals were dissolved in $\mathrm{CHCl}_{3}$ and $\mathrm{CDCl}_{3}$ for FT-IR and NMR analysis respectively. FT-IR which was recorded at room temperature shows the presence of $\mathrm{CH}=\mathrm{N}$ stretching (around $1636 \mathrm{~cm}^{-1}$ ) and aromatic $\mathrm{C}=\mathrm{C}$ (around $1595 \mathrm{~cm}^{-1}$ ). Proton NMR recorded for IMC shows peaks at $\delta(p p m)$ are $8.27(\mathrm{~s}, 6 \mathrm{H}), 7.66(\mathrm{~d}, \mathrm{~J}=7.9 \mathrm{~Hz}, 12 \mathrm{H})$, 7.45-7.35 (m, 9H), $7.02(\mathrm{dd}, J=8.5,13.4 \mathrm{~Hz}, 24 \mathrm{H}), 6.95-$ $6.90(\mathrm{~m}, 12 \mathrm{H}), 6.55(\mathrm{~s}, 3 \mathrm{H}), 1.64(\mathrm{~s}, 36 \mathrm{H})$. Corresponding Carbon NMR reveals peaks at $\delta$ (ppm) $158.5,150.1,149.4$, $148.5,148.4,131.8,131.1,130.2,127.4,124.0,122.0,120.0$, $42.8,30$.

\subsection{Macrocyclic system generation}

IMC system periodically arranged in a lattice with a unit cell of dimension $2 \times 2 \times 2$ with a molecule having centroid fit is shown in Fig. 2 . The whole system was subject to energy minimization by fixing the Lewis structure and connectivity using conjugate minimization. Orthorhombic periodic boundary condition in a vacuum with IMC system placed at the centre of mass was used as the starting structural assembly for all the molecular dynamics simulations.

\subsection{Thermophysical parameter analysis}

Theoretical glass transition temperature $T_{g}$ and coefficient of thermal expansion were determined based on volumetric residual density expansion of the unit cell at different temperature form $200-600 \mathrm{~K}$ by molecular dynamics simulation. An increment of $25 \mathrm{~K}$ was used to reach from 200 to $600 \mathrm{~K}$ and for each $25 \mathrm{~K}$ increment, a $20 \mathrm{~ns}$ simulation was carried out to understand the change in residual density. This type of in-depth simulation analysis for each step aid in understanding the stability of crystal packing thereby overall stability of cages in that particular packing

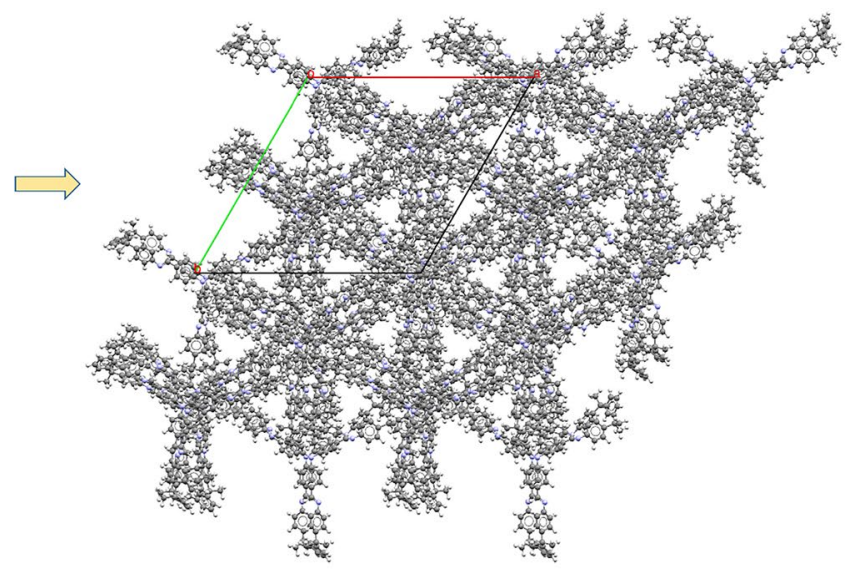

$2 \times 2 \times 2$ Unit cell with molecule having centroid fit.

SN Applied Sciences A SPRINGER NATURE journa 
arrangement. The value of $\mathrm{T}_{\mathrm{g}}$ and coefficient of thermal expansion are $444 \mathrm{~K}$ and $175.9510^{-6} \mathrm{~K}^{-1}$ at $298.15 \mathrm{~K}$, respectively. The result suggests a sudden increment in a specific volume of the system with heavy positional fluctuations at the temperature above $400 \mathrm{~K}$. Bilinear fit for IMC (MC-51) system as a function of temperature and specific volume is shown in Fig. 3 a.

\subsection{IMC's response to mechanical stress}

Systematic analysis of stress and strain was carried to measure the mechanical properties of the IMC in a periodic lattice arrangement. Huge discontinuous void spaces make IMC non-resilient to strain. Lower mechanical stability can be attributed as the consequence of greater residual voids in between the macrocyclic cage in the present arrangement. Chloroform removed structure was considered for calculations that would have reflected on the decreased stability. But the disordered nature of the chloroform $\left(\mathrm{CHCl}_{3}\right)$ itself explains the non-interacting behaviour of IMC with the solvent and hence the "stability by the solvent" factor is practically absent. Stress and strain scatter plot of the system is shown in Fig. 3b. The elastic anisotropy of the macrocycle system on X-axis is given in Fig. 3c.

\subsection{Physicochemical parameter analysis.}

The crystal structure was taken directly to analyse the ground state population energy. The orbital energy pattern shows the possibility of different absorption and excitation patterns corresponding to different electron delocalization (resonance) states. Except the phenyl ring attached with gem-dimethyl groups, all the other phenyl rings can assume resonance with the remaining structural part. Phenyl rings at the tertiary amine nitrogen (central
Nitrogen) which are twisted with one another from co-planarity and the possibility of rotation of the phenyl group adjacent to imine group might hinder the electron delocalization efficiency. The physicochemical parameters of IMC molecule were calculated with B3LYP 6-31 $(d, p)$ basis set to assess the orbital energy. The HOMO-LUMO orbital map of IMC with 4 delocalized states is shown in Fig. 4. The physicochemical parameters of IMC are given in Table 2 . Lower order photocatalytic band gap materials are essential in tailoring optical performance during engineering functional materials. IMC has the band order of $2.38 \mathrm{eV}$ [21] and has promising photoreceptive characteristics as photoabsorber.

\subsection{Intermolecular packing analysis.}

There are three molecules packed in the asymmetric unit. The molecules were clustered into three groups based on clustered energy. The packing lattice energy of the molecule of first three clusters are $122.1 \mathrm{~kJ} \mathrm{~mol}^{-1}, 119.1 \mathrm{~kJ} \mathrm{~mol}^{-1}$ and $30.7 \mathrm{~kJ} \mathrm{~mol}^{-1}$ respectively. The packing fraction with lattice energies is shown in Figure $\mathrm{S} 8$.

\section{Experimental section}

\subsection{Molecular crystallizations}

A solution of benzene trialdehyde $(0.010 \mathrm{~g}, 0.061 \mathrm{mmol})$ in acetonitrile (ACN) was slowly added to a solution containing gem-dimethyl amine $(0.032 \mathrm{~g}, 0.09 \mathrm{~mol})$ in ACN. $50 \mu \mathrm{l}$ of acetic acid was added to the ACN solution and was kept undisturbed for 7 days to obtain pale yellow colored, needle-shaped crystals. A detailed synthesis of the IMC, macrocycle 10 is as reported [22]
Fig. 3 Thermophysical and mechanical analysis of macrocyclic cage MC-51. a Thermophysical plot relating temperature with respect to specific volume, b Stress strain analysis, and c elastic property of macrocyclic cage MC-51

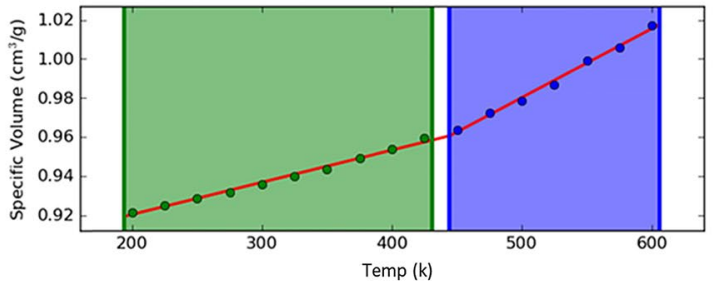

(a) Thermo-physical analysis
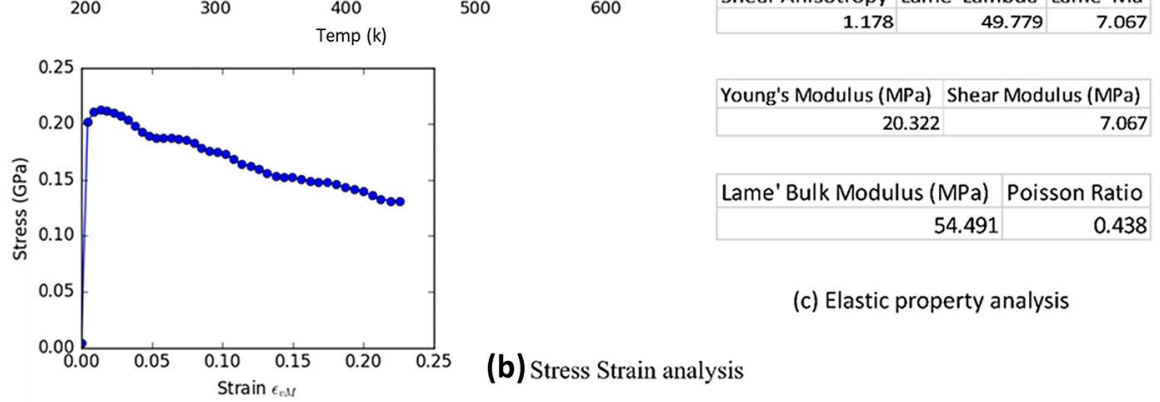

(c) Elastic property analysis

(b) Stress Strain analysis 
Fig. 4 Delocalized orbital plot of 4 HOMO-LUMO occupied state of isolated MC-51 molecule

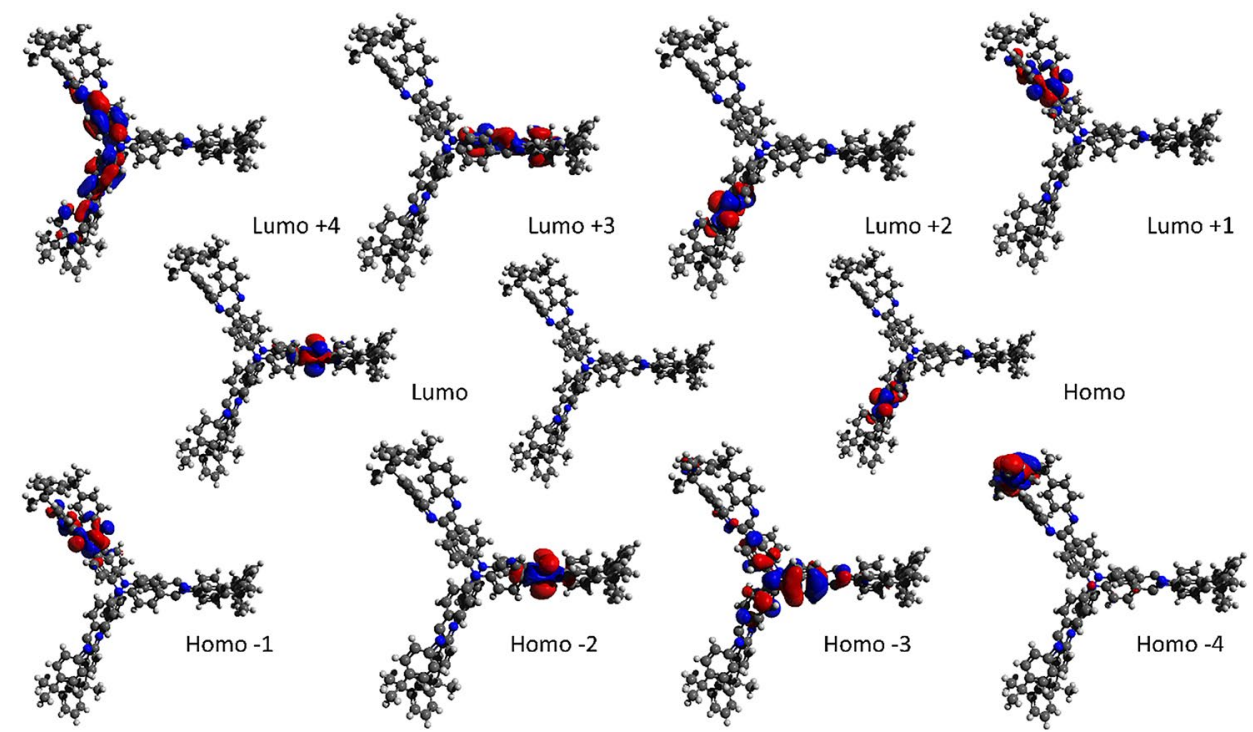

\subsubsection{X-Ray data collection and Refinement}

X-ray diffraction and intensity measurements of the gemdimethyl compound were obtained on Bruker SMART APEX CCD diffractometer with graphite-monochromatized $\left(\mathrm{MoK}_{\mathrm{a}}=0.71073 \AA\right)$ radiation. The X-ray data collection was monitored by the SMART program [23]. Lorentz factor, polarization and absorption of the crystal data were corrected using SAINT and SADABS programs. The initial structure was solved using SIR92 and further refinement was done using SHELXL [20] with full matrix least-squares refinement on structure factors. Geometrical packing analysis and their diagrams were generated using Mercury suite [24].

Initial structure refinements revealed the presence of chloroform in the lattice but were heavily disordered. The phenyl ring which links two gem-dimethyl methylene groups is also found disordered from planarity. To overcome these, squeeze procedure was carried by masking the regional density map keeping only the ordered macrocyclic structure and AFIX66 procedure was employed. Since the IMC system is in a disordered state, the hydrogen atoms were not assigned. In comparison with structures from $C C D C$, some deviations were observed among certain geometrical parameters. It has to be mentioned here that the IMC crystals were fragile and did not pose higher order stability.

\subsection{NMR and FT-IR analysis.}

NMR spectroscopic investigation was carried out to obtain chemical shift $\delta$ ppm in reference to TMS. IR spectroscopy was recorded using $\mathrm{CHCl}_{3}$ or Nujol on Bruker-FTIR spectrophotometer.

\subsection{Macrocyclic system generation.}

The Macrocyclic cages were periodically arranged to the lattice value of $2 \times 2 \times 2$ order with the connectivity of basis. The molecular system was minimized to correct all the Lewis structure and atomic bond orders. Polak-Ribiere Conjugate Gradient (PRCG) conjugate minimization was carried out with 10,000 maximum iteration steps with the convergence of $0.001 \mathrm{kcal} \mathrm{mol}^{-1} \AA^{-1}$. The system was prepared in the orthorhombic periodic boundary condition using system builder Materials science suite [25].

\subsection{Thermophysical analysis.}

Molecular dynamics simulation-based thermophysical properties calculation was done using Desmond [26]. The protocol of simulation involves system relaxation. The relaxation protocol involves minimization of the system for 100 ps Brownian dynamics at $10 \mathrm{~K}$ with $24 \mathrm{ps}$ simulation of NVT ensemble at $300 \mathrm{~K}$ and $700 \mathrm{~K}$, respectively with $1 \mathrm{~ns}$ equilibration at $300 \mathrm{~K}$ followed by $20 \mathrm{~ns}$ at a different temperature range from 200 to $600 \mathrm{~K}$ to evaluate the differential converged density of the system. The bilinear fit was performed to analyze the glass transition temperature $T_{g}$. The coefficient of thermal expansion was generated based on change in density with respect to temperature. 
Table 1 Crystal data and structure determination details

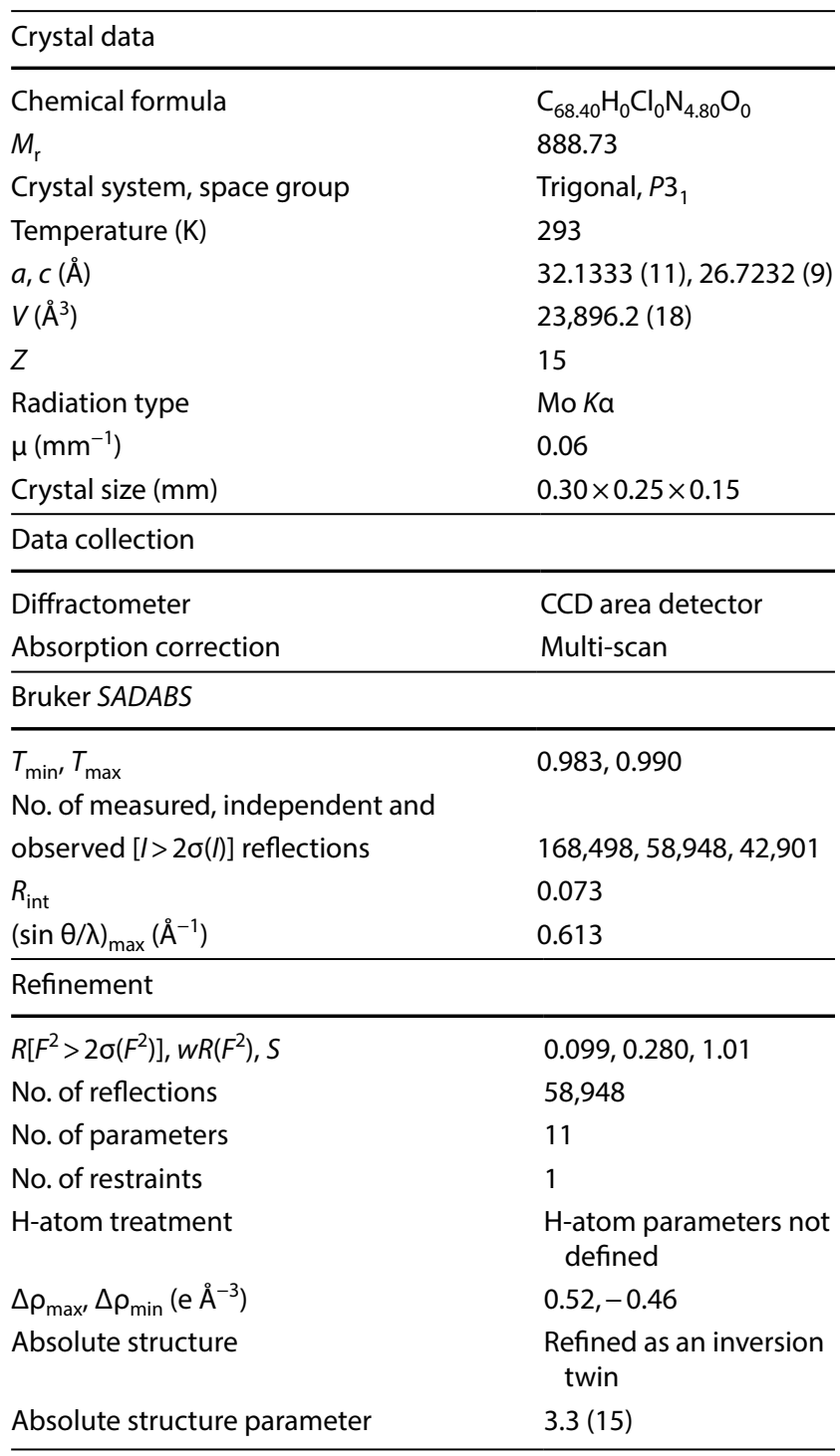

Computer programs: Bruker SMART [19], Bruker SAINT [19], SHELXL [20]

\subsection{Elastic constants evaluation of the mechanical response of the system}

The stress is evaluated by accounting change in pressure tensor from initial and final deformation using Desmond [26]. $20 \mathrm{~ns}$ molecular simulation was run to analyze the stress of the system as a function of strain. The system was run in NVT ensemble at $300 \mathrm{~K}$. The strain is determined based on parameter $\eta=0.5$ which is volume conserved uniaxial strain to relate the transverse strain $\lambda$.

$\lambda=1-(1+\varepsilon)^{-\eta}$

The strain is analyzed on X-axis based on fractional increment in uniaxial length given by equation.

$L_{K}=L_{0}(1+k \varepsilon)$

Elastic properties such as Tension anisotropy, Shear anisotropy, Lamé Lambda, Lamé Mu, Young's Modulus, Shear Modulus, Lamé Bulk Modulus, and Poisson Ratio were evaluated based on strain calculations.

\subsection{Physicochemical parameter determinations}

Crystal structure optimized with the adiabatic Born-Oppenheimer approximation using DFT B3LYP 6-31(d,p)[27] basis set till stationary potential state with lowest saddle point was found out with Berny algorithm by means of self-consistent field (SCF) geometry optimization with energy embodied direct inversion in the iterative subspace (GEDDIS) method [28]. The energy was analyzed based on ground state $\left(\mathrm{S}_{0}\right)$ derived orbital states. The orbital computations and optimization are done with Avogadro [29] and Gaussian 03, respectively[30].

\subsection{Intermolecular Interaction energy}

The intermolecular lattice energies were calculated using OPIX program [31, 32]. The summation of inter lattice energy is computed by UNI force fields where the pairwise atom-atom interactions are calculated. The overall intermolecular potential was calculated as the summation of UNI force field parameters consisting of coulombic, polarization, and dispersion and repulsion energy terms, respectively. Intermolecular potential associated with centrosymmetric assembly can also be identified.

Table 2 Physicochemical parameters of IMC molecule

\begin{tabular}{|c|c|c|c|c|c|c|c|c|}
\hline $\begin{array}{l}\text { Compound } \\
\text { Name }\end{array}$ & $\begin{array}{l}\text { lonisation } \\
\text { potential(I) } \\
(\mathrm{eV})\end{array}$ & $\begin{array}{l}\text { Electron affin- } \\
\text { ity }(\mathrm{A})(\mathrm{eV})\end{array}$ & $\begin{array}{l}\text { gap energy, } \\
\Delta \mathrm{E}\end{array}$ & $\begin{array}{l}\text { Chemical } \\
\text { Hardness( } \eta)\end{array}$ & Softness(S) & $\begin{array}{l}\text { Chemical } \\
\text { Potential }(\mu)\end{array}$ & $\begin{array}{l}\text { Electronega- } \\
\text { tivity }(\chi)\end{array}$ & $\begin{array}{l}\text { Electrophilicity } \\
\text { index }(\omega)\end{array}$ \\
\hline MC-51 & 5.80 & 3.42 & 2.38 & 1.19 & 0.84 & -4.61 & 4.61 & 8.92 \\
\hline
\end{tabular}




\section{Conclusions}

Self-assembling macrocyclic cages has wide ranging applications from energy storage to photoabsorber. Here self-assembling gem-dimethyl frameworks was constructed and spectral and crystallographic investigation were carried out. The major stabilizing force of crystal packing are $\pi-\pi$ intermolecular networks between phenyl groups also observed from molecular dynamics simulations. Elastic, thermophysical, mechanistic properties also were computed to analyse the stability of the imine based macrocyclic molecular geometry. The gemdimethyl group tends to be moderately stable and crystal system can withstand intermediate mechanical stress. It has similar band gap value to that of photocatalytic materials, and indicates suitability in constructing MOF's with catalytic applications. These cages has to be further be incorporated with MOF's to engineer functional materials having catalytic and gas storage applications.

Acknowledgements SS thanks Schrodinger for Desmond and Material science studio licence. MS is thankful to CSIR-UGC, New Delhi for research fellowship. Authors also thank CDAC-BRAF facility for Gaussian 03 program.

\section{Compliance with ethical standards}

Conflict of interest Authors declare no conflict of interest.

Open Access This article is licensed under a Creative Commons Attribution 4.0 International License, which permits use, sharing, adaptation, distribution and reproduction in any medium or format, as long as you give appropriate credit to the original author(s) and the source, provide a link to the Creative Commons licence, and indicate if changes were made. The images or other third party material in this article are included in the article's Creative Commons licence, unless indicated otherwise in a credit line to the material. If material is not included in the article's Creative Commons licence and your intended use is not permitted by statutory regulation or exceeds the permitted use, you will need to obtain permission directly from the copyright holder. To view a copy of this licence, visit http://creativecommons .org/licenses/by/4.0/.

\section{References}

1. Liu Z, Nalluri SKM, Stoddart JF (2017) Surveying macrocyclic chemistry: from flexible crown ethers to rigid cyclophanes. Chem Soc Rev 46:2459-2478. https://doi.org/10.1039/c7cs0 0185a

2. Huang S-L, Jin G-X, Luo H-K, Hor TSA (2015) Engineering organic macrocycles and cages: versatile bonding approaches. Chem - An Asian J 10:24-42. https://doi.org/10.1002/ asia.201402634

3. Cooper Al (2017) Porous molecular solids and liquids. ACS Cent Sci 3:544-553. https://doi.org/10.1021/acscentsci.7b00146

4. Tashiro S, Kubota R, Shionoya M (2012) Metal-Macrocycle framework (MMF): Supramolecular Nano-Channel Surfaces with shape sorting capability. J Am Chem Soc 134:2461-2464. https://doi.org/10.1021/ja209422m
5. Jiang S, Jones JTA, Hasell T et al (2011) Porous organic molecular solids by dynamic covalent scrambling. Nat Commun 2:207. https://doi.org/10.1038/ncomms 1207

6. Schouwey C, Scopelliti R, Severin K (2013) An imine-based molecular cage with distinct binding sites for small and large alkali metal cations. Chem - A Eur J 19:6274-6281. https://doi. org/10.1002/chem.201300098

7. Liao M-S, Scheiner $S$ (2002) Electronic structure and bonding in metal porphyrins, metal=Fe Co, Ni, Cu, Zn. J Chem Phys 117:205-219. https://doi.org/10.1063/1.1480872

8. Wilkinson F, McGarvey DJ, Olea AF (1994) Excited triplet state interactions with molecular oxygen: influence of charge transfer on the bimolecular quenching rate constants and the yields of singlet Oxygen [O*2(1.DELTA.g)] for substituted naphthalenes in various solvents. J Phys Chem 98:3762-3769. https://doi.org/10.1021/j100065a035

9. Schweitzer C, Mehrdad Z, Noll A et al (2003) Mechanism of photosensitized generation of singlet oxygen during oxygen quenching of triplet states and the general dependence of the rate constants and efficiencies of $\mathrm{O}_{2}(1 \Sigma \mathrm{g}+), \mathrm{O}_{2}(1 \Delta \mathrm{g})$, and $\mathrm{O}_{2}(3 \Sigma \mathrm{g}-)$ formation on sensitizer triplet St. J Phys Chem A 107:2192-2198. https://doi.org/10.1021/jp026189d

10. Zhu R, Ding J, Jin L, Pang H (2019) Interpenetrated structures appeared in supramolecular cages, MOFs, COFs. Coord Chem Rev 389:119-140. https://doi.org/10.1016/j.ccr.2019.03.002

11. Chen S, Li K, Zhao F et al (2016) A metal-organic cage incorporating multiple light harvesting and catalytic centres for photochemical hydrogen production. Nat Commun 7:13169. https://doi.org/10.1038/ncomms13169

12. Leenders SHAM, Gramage-Doria R, de Bruin B, Reek JNH (2015) Transition metal catalysis in confined spaces. Chem Soc Rev 44:433-448. https://doi.org/10.1039/C4CS00192C

13. Juse'lius J, Sundholm D, (2000) The aromatic pathways of porphins, chlorins and bacteriochlorins. Phys Chem Chem Phys 2:2145-2151. https://doi.org/10.1039/b000260g

14. Hasselman GM, Watson DF, Stromberg JR et al (2006) Theoretical solar-to-electrical energy-conversion efficiencies of perylene-porphyrin light-harvesting arrays $\dagger$. J Phys Chem $B$ 110:25430-25440. https://doi.org/10.1021/jp064547x

15. Cao Y, Fatemi V, Fang $S$ et al (2018) Unconventional superconductivity in magic-angle graphene superlattices. Nature 556:43-50. https://doi.org/10.1038/nature26160

16. Liu W, Liu J, Miao M (2016) Macrocycles inserted in graphene: from coordination chemistry on graphene to graphitic carbon oxide. Nanoscale 8:17976-17983. https://doi.org/10.1039/ C6NR04178G

17. Desiraju GR (2013) Crystal engineering: from molecule to crystal. J Am Chem Soc 135:9952-9967. https://doi.org/10.1021/ ja403264c

18. Hooley RJ (2018) Rings and things: the magic of building selfassembled cages and macrocycles. Inorg Chem 57:3497-3499. https://doi.org/10.1021/acs.inorgchem.8b00553

19. Bruker AXS (2008) APEX2, V2008. 6, SADABS V2008/1, SAINT V7. 60A, SHELXTL V6. 14. Bruker AXS Inc, Madison, Wisconsin, USA

20. Sheldrick GM (2015) Crystal structure refinement with SHELXL. Acta Crystallogr Sect C Struct Chem 71:3-8. https://doi. org/10.1107/S2053229614024218

21. Wu A, Li J, Liu B et al (2017) Band-gap tailoring and visible-lightdriven photocatalytic performance of porous $(\mathrm{GaN}) 1-\mathrm{x}(\mathrm{ZnO}) \mathrm{x}$ solid solution. Dalt Trans 46:2643-2652. https://doi.org/10.1039/ C6DT04428J

22. Madhu S, Rashmi EV, Gonnade RG, Sanjayan GJ (2017) Exploring the gem-dimethyl effect in the formation of imine-based macrocycles and cages. New J Chem 41:8721-8724. https://doi. org/10.1039/C7NJ02069D 
23. Bruker A (2012) APEX, APEX2, SMART, SAINT, SAINT-Plus. Bruker (2012) Progr name(s) Bruker AXS Inc, Madison, Wisconsin, USA [Older versions should Ref to Siemens Anal X-ray Instruments Inc instead Bruker AXS]

24. Macrae CF, Sovago I, Cottrell SJ et al (2020) Mercury 4.0: from visualization to analysis, design and prediction. J Appl Crystallogr 53:226-235. https://doi.org/10.1107/S1600576719014092

25. Suite MS, (2018) Materials Science Suite 2018-4. Schrödinger, LLC, New York, NY, p 2018

26. (2006) SC '06: Proceedings of the 2006 ACM/IEEE Conference on Supercomputing. ACM, New York, NY, USA

27. Tirado-Rives J, Jorgensen WL (2008) Performance of B3LYP density functional methods for a large set of organic molecules. $J$ Chem Theory Comput 4:297-306. https://doi.org/10.1021/ct700 248k

28. Frisch MJ (2006) Li_Frisch_jctc2006. 835-839. doi: https://doi. org/10.1021/ct050275a

29. Hanwell MD, Curtis DE, Lonie DC et al (2012) Avogadro: an advanced semantic chemical editor, visualization, and analysis platform. J Cheminform 4:17. https://doi. org/10.1186/1758-2946-4-17

30. Frisch MJ, Trucks GW, Schlegel HB, et al Gaussian 03, Revision C.02

31. Gavezzotti A (1994) Are Crystal Structures Predictable? Acc Chem Res 27:309-314. https://doi.org/10.1021/ar00046a004

32. Gavezzotti A, Filippini $G$ (1994) Geometry of the intermolecular $X-H . c n t d o t . c n t d o t . c n t d o t . Y(X, Y=N, O)$ hydrogen bond and the calibration of empirical hydrogen-bond potentials. J Phys Chem 98:4831-4837. https://doi.org/10.1021/j100069a010

Publisher's Note Springer Nature remains neutral with regard to jurisdictional claims in published maps and institutional affiliations. 